Verfahrens außer Zweifel. Desgleichen kann angenommen werden, daß das direkte Hinausschieben des Blockes aus der Aufschließkammer, d a s, ganz abgeseben vonder einfachen Arbeitsweise, die Anlagevon Wagen und Geleisen entbehrlich macht, unterbestimmten Arbeitsverhält. nissen einen technischen Fortschritt bildet."

Ich glaube, daß diese beiden Entscheidungen rollkommen genügen, und ich brauche nur wenig hinzuzufügen.

Es dürfte jetzt wohl deutlich sein, daß anstatt ein Rückschritt, wie Herr Beskow behauptet, diss Svenskaverfahren einen bedeutenden Fortschritt bildet. Den kühnen Schritt, den die Erfinder des Systems Svenska machten, als sie, auf die genaucsten, theoretischen Berechnungen gestüt $z$, ohne weiteres ihre Idee im großen Maßstabe ausführten und ein durchaus glänzendes Resultat erreicliten, nenne ich bewundernswert. Sie machten sich von allen früher vorgeschlagenen und ausgefiihrten mehr oder weniger komplizierten Ausweren, um die Reaktionskammern mechanisch zu entleeren, vollkommen frei und schufen eine Entlerrungsmethode, welche betreffs Einfachheit und dimit zusammenhängender Betriebssicherheit alle anderen Systeme weit hinter sich läBt.

Ihie Behauptung des Herrn Besk ow betreffend, daß die Malmöer Fabrik nur citratlösliche's Superphosphat herstellt, sei hier noch erwähnt, daß ein großer Teil der Produktion dieser Fabrik verkauft wird nach Ländern, welche nur wasserlösliche Phosphorsäure gebrauchen: es dürfte diese Tatsache Herrn Bes kow sehr wohI bekinnt sein.

Was die Behauptung anbelangt, daß ,Svenska“" gewissermaßen ein liegender $\mathrm{S}$ c h $\mathrm{u} \mathrm{ch}$ t scher $\mathrm{Ap}$ parat sei, so braucht doch wirklich nicht bewiesen zu werden, daß zwei Apparate, wovon der eine zum Zwecke der Herstellung einer kräftigen Zusammen. pressung und der andere zum Zweok der Vermeidung einer solchen Zusammenpressung konstruiert wurden, von ganz entgegengresetzten J'rinzipien sind!

Ich freue mich, inzwischen der deutschen und österreichischen Düngerindustrie nunmehr (ielegenheit geben zu kïnnen, sich von der wirtschaftlichen Bedeutung des .. Krensika". Verfahrens überzeugen zu könurn.

[A. 26.

\section{Zusätze für die Wasserreinigung II.} Von Dr. E. F. Basch.

(Fingeg. 17.|2. 1911.)

Die Bemerkungen von Herrn Dr. H. Noll in dieser Z. 24, 208 (1911), die sich mit meinem vorangegangenen dufsat $\%$ in dieser $Z$. 23, 2205 (1910) befassen, veranlassen !nich zu nachstehender Entgegnung.

Die Restharten des gereinigten Wassers kamn ich unmöglich als $x$ alciumcarbonat aufgefaßt haben, denn in den drei von mir zitierten Beispielen wurden ersichtlicherweise die Summanden Kalkhärte und Magnesiahärte gesundert mit ihrem jeweiligen ZahJenwerte eingesetzt. Aus don angeführten Gesamt- härten habe ich dann gefolgert, daß ,eine Enthärtung auf $1^{\circ}$ deutsch und weniger ganz ausgeschlossen" sei, worauf es mir zur Widerlegung der gegenteiligen Ansicht von Dr. Drawe (diese Z. 23, 52 [1910]) im besonderen ankam.

DaB die Löslichkeit von Calciumcarbonat in "Salzlösungen" wesentlich geringer ist als im dest. Wasser, bezweifle ich selbstverständlich nicht. Die erste diesbezügliche Anmerkung von Herrn Dr. No 11 in dieser Z. 23, $1463(1910)$ bezog sich jedoch nicht auf theoretisch mögliche Fälle, sondern auf die für die Praxis angegebenen Formeln von Dr. $\mathrm{R}$ is te $n$ part (diese Z. 23, 394 [1910]). In der Praxis kann aber zugegebenermaßen nicht mit 50\% SodaüberschuB gerechnet werden; das ist somit ein theoretischer Fall. In meiner Bemerkung: „Die von ihm (Dr. $\mathrm{Noll}$ ) beim Hamburger Leitungswasser erhaltenen und oben zitierten Zahlen stützen seine Vermutung allerdings nicht ...", sind die Worte „oben zitierte" nnchträglich und irrtümlich hineingeraten. Die von mir angefïhrte, auch theo. retische Gipslösung mit überschüssigem Calciumsulfat von ca. $100 \mathrm{FH}$ war absichtlich als Lehr. beispiel gewählt, um zı zeigen, daß selbst bei cinem übertrieben hohen Salzgehalt die Löslichkeit von Calciumcarbonat noch beträchtlich ist.

Herr Dr. Xoll macht darauf aufmerksam, daB jene mit ungenügendem Sodazusatz versehene Gipslösung beim Kochen nach einer Viertelstuncle wieder eine deutliche Phenolphthaleinrötung annimmt. Diese Beobachtung - die an der Sache selbst nichts ändert - habe ich bei der Nachpriifung bestätigt gefunden. Die von mir übersehene Anwesenheit von etwas gelöstem Calciumbicarbonat führe ich auf die verwendete abgestandene Sodalösung zurück. Die durch Auskochen erhaltene Rotfärbung verschwindet aber schon beim zweiten Tropfen $1 / 1^{-n}$. Säure. Daß Calciumcarbonat Phenolphthalein ,ebensogut" "rötet wie Sodalösung, ist wohl nur im qualitativen Sinne von .,auch" rötet gemeint, denn quantitativ ist ein großer lnterschied vorhanden. Gelöstes Xatriumearbonat wird bekanntlich durch Phenolphthalein im lalben Betrage seiner Gesamtalkalit it angezeigt, so da .3 man sich in verd. lösung diese Dissoziaton vorstellen kann:

$$
\mathrm{Na}_{2}\left(\mathrm{O}_{3} \cdot \mathrm{H}_{2} \mathrm{O}:=\mathrm{XaH}_{\mathrm{a}} \mathrm{HCO}+\mathrm{NaOH}\right. \text {. }
$$

Nicht so beim ('alciumcarbonat! Ich habe solches zunäichst mit dest. Wasser unter Zugabe von etway Kalkwasser ausgekocht, um dadurch mit Niche:'heit etwaiges (alciumbicarbonat auszuselualten. Nach dem Filtrieren und violligen Auswaschen habe ich den Löslichkeitsversuch von Dr. Noll wiederholt und für je 200 ccm Flüssigkeit verbratueht: im Waschwasser in der Lüsung mit Phenolphthalein $1,6 \mathrm{~cm} \quad 0,1 \frac{1}{1} / 0^{-n}$. H(l. mit Methylorange $\quad 2,6$,

\section{Über Konzentration von Schwefel- säure auf $97-98 \%$ Monohydrat.}

Berichtigung zu dem Aufzatz von A. G. Dtrox von G. Stolzf.rwalo.

(Eingeg. d. 20.2.2. 1911.)

Erst heute kommen mir die Bemerkung('n von A. G. D u r on in dieser $Z .23,2307$ (1910) ïlor 
„Konzentration von Schwefdeäure auf 97-98\% Monohydrat" zu Gesioht, welche meine in dem Aufsatz in dieser Z. 23, 1975 (1910) vertretenen Ansichten in ihr Gegenteil verkehren. D u r o $n$ behauptet, ich bätte ausgeführt, da $B$ eine ökonomische Konzentration nur in einer kombinierten Anlage, bestehend aus Vorkonzentration und Fertigverdampfer, möglich sej, und daB dieses auch ohne Sehwierigkeit auf direktem Wege mit dem Apparat von $G$ a illa $\mathrm{r} d$ und dem von $\mathrm{K}$ e $\beta \mathrm{l}$ e $\mathrm{r}$ erreicht werden kann. Aber gerabe diese zwei genannten Apparate habe ich mit „Vollapparate" bezeichnet, weil in ihnen allein die Säure zur höchsten Konzentration gebracht werden kann, und weiter ausgeführt, daß im Gegensatz zu den Vollapparaten die kombinierten Verfahren stehen, weil diese eine größere Auswahl von Apparaten zur Verfügung stellen.

Die Behauptungen $\mathrm{D}$ u r o $\mathrm{n}$ s entbehren daher jedweder Grundlage.

[A. 38.]

\section{Zwei Gedächtnisregeln.}

\section{Von E. H. Riesenfeld.}

(Eìngeg. 18.2. 1911.)

Aus einer Bemerkung, die Herr Prof. Bött . ge $r$ in seinem Referat uber mein „AnorganischChenisches Praktikum" machtel), ersehe ich, da $B$ einige der kleinen (iedächtnisregeln, die ich darin zusammenstellte, nicht so bekannt sind, wie sie es vielleicht verdienton. Ioh möchte sie daher hior mitteilen. Sio betreffen eine, rein z u fällige Beziehung zwischen Prozentgehalt, spez. Gew. und Molarität von Säuren. Mit Prozentgehalt wird hierbei in der üblichen Weise die Anzahl der g Säure bezejchnet, die in $100 \mathrm{~g}$ lösung enthalten sind, und mit Molarität die $g$ Säure, die in 11 gelöst sind.

1. Der Prozentgehalt von konz. Salz-, Salpeterund Schwefelsäure ist gleich ihrem Molargewicht, ihre Molarität das Zehnfache ihres spez. Gew.

$$
\text { Beweis : }
$$

$\begin{array}{lccccc}\text { konz. } & \begin{array}{c}\text { Baumé } \\ \sigma\end{array} & \begin{array}{c}\text { Molar- } \\ \text { gow. }\end{array} & \begin{array}{c}\text { Gehalt } \\ \%\end{array} & \begin{array}{c}\text { spez.Ge- } \\ \text { wicht }\end{array} & \begin{array}{c}\text { Molari } \\ \text { tat }\end{array} \\ \mathrm{HCl} \cdot \cdot 23 & 36 & 37 & 1,2 & 12 \\ \mathrm{HNO}_{2} \cdot 40 & 63 & 63 & 1,4 & 14 \\ \mathrm{H}_{2} \mathrm{SO}_{4} \cdot 66 & 98 & 98 & 1,8 & 18\end{array}$

2. Der Prozent́gehalt (p) wässeriger salzsäurelösungen ist das 200 fache der Differenz ihres spez. Gew. (s) und desjenigen des Wassers, also

$$
\mathrm{p}=200(\mathrm{~s}-1) \text {. }
$$

Beweis:

$\begin{array}{cccc}\text { spez. Gew. } & \begin{array}{c}\text { Gehalt } \\ \%\end{array} & \text { spez. Gew. } & \text { Gehalt } \\ 1,01 & \% & 1,10 & \% \\ 1,05 & 10 & 1,15 & 30\end{array}$

[A. 36.]

\section{Wirtschaftlich-gewerblicher Teil.}

\section{Jahresberichte der Industrie und des Handels.}

Philippinen. Nach einem Berichte des Kaiserl. Konsulates in Manila befindet sich die $\mathrm{K}$ o p r a. in $\mathbf{d ~ u s} \mathrm{t} r$ i e auf den Philippinen in aufblühendern Zustande. In allen Provinzen des Insellandes vermehren sich die Anpflanzungen der Cocosnußpalme von Jahr zu Jahr in bedeutendem Maße. Die philippinische Kopragewinnung betrug 1907: 844909 , 1908: 1345 166, 1909: 1658724 Pikuls (zu 63 kg), der Wert der Ausfuhr von Kopra in den entspechenden Jahren 4784 151, 6058886 und 7672865 Doll. Der Preis für Kopra ist infolge der gesteigerten Nachfrage nach riesem wertvollen Fet $t$ stoffe sehr gestiegen. Die vermehrte Nachfrage ist namentlich auf das Anwachsen der Preise für tie. rische Fette, wie auch für das als Ersatz für Schweinefett viel gelorauchte Baunwollensaatö] zu rückzuführen. Auch als Ersatz für Talg und Butter kommt Kopra immer mehr in Gebrauch, und Cocosnußöl findet weitgehende Verwertung in den verschiedensten Industrien. -l. [K. 151.)

Japan. Hiner vom Ministerium für Ackerbau und Handel herausgegebenen Statistik sind folgende Zahlen über die Frzeugung oder Förderung mineralischer Stoffe i. J. 1909 (1908) entnommen; die Zahlen geben die Werte in $1000 \mathrm{Y}$ en

1) Jiese Z. 24, 20 (191I). an: Gold 5077 (4457), Silber $426 \mathrm{l}$ (4362), Kupfer 24411 (22 409), Blei 428 (405), Zinn 28 (35), Antimon 39 (53). Zinkerz 478 (308), Gußeisen 2025 (1736), Schmiedeeisen 30 (9), Stahl 469 (208), Eisenpyrite 106 (171), Chromeisenerz 27 (13), Manganerz 51 (82), Graphit 10 (17), Kohle 58211 (63 623), Torf 177 (144), Petroleum (j428 (6520), Asphalt 90 (51), Schwefel $812(748)$, inggesamt (einschl. anderer, nicht genannter) 103200 (105393).

$$
- \text { l. [K. 125.] }
$$

Britiseh-Indien. Utber die Entwicklung des Bergbaues in Britisch. Indien bringt der vor kurzem erschienene 39. Band der Veröffentlichungen der Geologischen Landesanstalt in Indien wichtige Angaben, die die Ansicht begründet erscheinen lassen, daß sich dem Bergbau in Indien noch weite Aussichten bieten dürften. Die Angaben beziehen sich auf den Abbau von 16 Mineralien, sie lassen den Fortschritt erkennen, der während der letzten 5.Berichtsjahre $(1904-1908)$ zu verzeichnen ist. Denn während der durchschnittliche Wert sich für die 5 Vorjahre noch auf ungefähr $88^{3} / 4$ Mill, M im Jahre stellte, betrug er für die Zeit von 1904 bis 1908 bereits $137 \frac{1}{2}$ Mill. M. Wird das Jahr 1908 allein in Betracht gezogen, so bewertete sich die Förderung sellust noch um etwa 20 Mill. M mehr. Kennzeichnend für Indien ist, daB anch jetzt (wie in früheren Jahren) nur eine vermehrte Tätigkeit in der Förderung soleher Mineralien festzustellen ist, die entweder einen unmittelbaren 